\title{
Nickel(II) Salicylaldiminates: Re-visiting a classic ${ }^{\#}$
}

Supaporn Saechio, ${ }^{1}$ Rodolphe Clérac, ${ }^{2}$ Keith S. Murray, ${ }^{3}$ Wasinee Phonsri, ${ }^{3}$ Eliseo Ruiz, ${ }^{4}$ Phimphaka Harding ${ }^{1^{*}}$ and David J. Harding ${ }^{1^{*}}$

${ }^{1}$ Functional Materials and Nanotechnology Center of Excellence, Walailak University, Thasala, Nakhon Si Thammarat, 80160, Thailand

2 Univ. Bordeaux, CNRS, Centre de Recherche Paul Pascal, UMR 5031, 33600 Pessac, France

${ }^{3}$ School of Chemistry, Monash University, Clayton, Melbourne, Victoria, 3800, Australia

${ }^{4}$ Departament de Química Inorgànica i Orgànica and Institut de Recerca de Química Teòrica i Computacional, Universitat de Barcelona, Diagonal 645, 08028 Barcelona, Spain

E-mail: kphimpha@mail.wu.ac.th and hdavid@mail.wu.ac.th

\# We dedicate this paper to Professor R. H. Holm who passed away on $15^{\text {th }}$ February 2021. Dick was a pioneer in modern inorganic and bioinorganic chemistry with early interests in d-block Schiff Base chemistry.

\begin{abstract}
A series of $\mathrm{Ni}(\mathrm{II})$ trinuclear complexes, $\left[\mathrm{Ni}_{3}(\text { sala-X })_{6}\right]$ (sala-X $=0-[(p-\mathrm{X}$-phenylimino) methyl]phenol; $\mathrm{X}=\mathrm{Br}$ 1-Br, $\mathrm{Me}$ 1-Me and $\mathrm{OMe}$ 1-OMe), have been prepared and fully characterised. X-ray crystallographic studies reveal that the complexes are composed of three face-sharing octahedral $\mathrm{Ni}$ (II) metal ions bridged by the phenoxide oxygens of the sala-X ligands. Magnetic studies indicate that the magnetic $\mathrm{Ni}(\mathrm{II})$ centres are ferromagnetically coupled with the substituent group having only a minor impact on the magnitude of coupling. DFT calculations also support ferromagnetic coupling with smaller $\mathrm{Ni}-\mathrm{O}-\mathrm{Ni}$ angles leading to slightly larger coupling constants in line with previous studies.
\end{abstract}

Keywords: nickel complexes; X-ray crystallography; magnetic properties; DFT calculations

\section{Introduction}

Schiff bases are common ligands in coordination chemistry largely due to their ease of synthesis and ready modification. ${ }^{1}$ One of the most studied of the Schiff bases are salicylaldimines, typically prepared by the reaction of salicylaldehyde and a primary amine. Aryl amines are particularly diverse and their $\mathrm{Ni}(\mathrm{II})$ complexes have received considerable attention in the area of olefin oligomerisation and polymerisation. ${ }^{2-9}$ The complexes have the general 
formula $[\mathrm{Ni}(\operatorname{sala}-\mathrm{X})(\mathrm{R})(\mathrm{L})]$ and the tunability of both the phenyl and salicylaldimine rings permits substantial modification of catalytic behaviour.

In contrast, the simple [Ni(sala-X $)_{2}$ ] complexes were first reported in $1962^{10}$ and over the course of a decade their basic spectroscopic data were collected and reported. ${ }^{11-13}$ The pattern of substitution on the phenyl ring was found to be important with ortho-substituted complexes being diamagnetic in solution while para- or meta-substituted systems were paramagnetic. At the time, this was interpreted as the ortho complexes being square planar, while the other complexes were thought to associate in solution. The nature of the association was unclear, and although [ $\mathrm{Ni}\left(\right.$ sala-Me ${ }_{2}$ ] was reported to display a trinuclear octahedral structure in the solid state ${ }^{14}$ the structure is not present in the CSD. In seeking to definitively answer the question of the structure of these complexes, we have prepared $\left[\mathrm{Ni}_{3}(\text { sala- } \mathrm{X})_{6}\right]$ (sala-X $=0-[(p-\mathrm{X}-$ phenylimino)methyl]phenol; $\mathrm{X}=\mathrm{Br}$ 1-Br, Me 1-Me and OMe 1-OMe) determined their structures and explored their magnetic behaviour, with the results supported by DFT calculations.

\section{Experimental}

\section{General Remarks}

All compounds were prepared with reagent-grade solvents. All other chemicals were purchased from Sigma-Aldrich or TCI Chemicals and used as received. Hsala-X were prepared according to literature procedure ${ }^{10}$ using diisopropylether instead of $\mathrm{MeOH}$ as the solvent.

\section{Synthesis of $\left[\mathrm{Ni}_{3}(\mathrm{sala}-\mathrm{X})_{6}\right]$}

All complexes were synthesized according to the following general procedure:

To a solution of Hsala-X (2 mmol) in tetrahydrofuran $(10 \mathrm{~mL})$ was added NaOMe $(0.1082 \mathrm{~g}, 2$ $\mathrm{mmol}$ ) and methanol $(1 \mathrm{~mL})$ to increase solubility. $\mathrm{NiCl}_{2} \cdot 6 \mathrm{H}_{2} \mathrm{O}(1 \mathrm{mmol})$ was added and the solution was stirred overnight. The solution was evaporated to ca. $2 \mathrm{~mL}$ and filtered through a Buchner funnel. The solid was washed with ethanol $(10 \mathrm{~mL})$ and then recrystallized from $\mathrm{CH}_{2} \mathrm{Cl}_{2}$ /hexane giving the compounds as green crystalline solids.

\section{[Ni $\left.\mathrm{Ni}_{3}(\mathrm{sala}-\mathrm{Br})_{6}\right]$ 1-Br}

Yield 49\%. IR (cm $\left.{ }^{-1}\right)$ : 3022(m), 2899(m), 1610(sh). UV-Vis $\left(\varepsilon, \mathrm{M}^{-1} \cdot \mathrm{cm}^{-1}\right) 629(123)$.

\section{[ $\mathrm{Ni}_{3}(\text { sala-Me) })_{6}$ 1-Me}

Yield 29\%. IR (cm $\left.{ }^{-1}\right): 3001(\mathrm{~m}), 2938(\mathrm{~m}), 1615(\mathrm{sh})$. UV-Vis $\left(\varepsilon, \mathrm{M}^{-1} \cdot \mathrm{cm}^{-1}\right) 600(212)$.

\section{[ $\mathrm{Ni}_{3}(\text { sala-OMe) })_{6}$ 1-OMe}

Yield 23\%. IR (cm $\left.{ }^{-1}\right)$ : 3002(m), 2833(m), 1614(sh). UV-Vis $\left(\varepsilon, \mathrm{M}^{-1} \cdot \mathrm{cm}^{-1}\right) 615(142)$. 


\section{Spectroscopic studies}

Infrared spectra were recorded on a Bruker Tensor 27 FT-IR spectrometer with OPUS data collection program in the range of $400-4000 \mathrm{~cm}^{-1}$ (as KBr discs). UV-Vis spectra were recorded in Acetone, DCM and DMF at room temperature on an Avantes Fiber Optics Spectrometer with Avalight-DHC and Avaspec ULS2048XL-USB2 in the range of 200-1000 nm.

\section{X-ray crystallographic studies}

Crystallographic data for the basic complexes were collected using a Rigaku SuperNova diffractometer. The data were scaled, integrated and an absorption correction applied in CrysAlisPro. The structures were solved in SHELXT ${ }^{15}$ using intrinsic phasing and refined by full matrix least squares minimisation on $F^{2}$ using SHELXL. ${ }^{16}$ All non-hydrogen atoms were refined anisotropically. Hydrogen atom positions were calculated geometrically and refined using the riding model. All pictures were generated with OLEX ${ }^{2} .{ }^{17}$ Crystallographic data for the structures have been deposited with the Cambridge Crystallographic Data Centre, with the CCDC numbers 2079808-2079810 for 1-Br to 1-OMe, respectively.

\section{Magnetic Studies}

Data on 1-Br and 1-OMe were collected with a Quantum Design MPMS 5 SQUID magnetometer under an applied field of $1 \mathrm{~T}$ or $0.1 \mathrm{~T}$ over the temperature range 2-300 $\mathrm{K}$. The powdered or polycrystalline samples were placed in gel capsules and care was taken to allow long thermal equilibration times at each temperature. DC magnetic susceptibility measurements on 1-Me were carried out with a Quantum Design SQUID magnetometer MPMS-XL. This magnetometer works between 1.8 and $400 \mathrm{~K}$ for dc applied fields ranging from -7 to $7 \mathrm{~T}$. Ac susceptibility measurements were performed with a Quantum Design PPMS-9 susceptometer working with an oscillating ac fields of 1 up to 10 Oe with a frequency between 10 and $10000 \mathrm{~Hz}$. Consistent dc susceptibility at $0.1 \mathrm{~T}$ and in-phase ac susceptibility in zero-dc field have been obtained between 1.85 and $15 \mathrm{~K}$ without detection of an out-of-phase ac signal up to $10000 \mathrm{~Hz}$, up to $2.8 \mathrm{~T}$ and above $1.9 \mathrm{~K}$. These magnetic measurements were performed on a microcrystalline sample (13.58 $\mathrm{mg}$ ) sealed in a polyethylene bag $(3 \times 0.5 \times 0.02 \mathrm{~cm}$; typical mass of $12.37 \mathrm{mg})$ and restrained in mineral oil $(6.78 \mathrm{mg}$ ). Magnetic data were corrected for the sample holder, mineral oil and diamagnetic contributions.

\section{Results and Discussion}

\section{Synthesis, structure and spectroscopic characterizations}

The compounds $\left[\mathrm{Ni}_{3}(\text { sala- } \mathrm{X})_{6}\right](\mathrm{X}=\mathrm{Br}$ 1-Br, Me 1-Me and OMe 1-OMe) were synthesized by reacting $\mathrm{NiCl}_{2} \cdot \mathrm{H}_{2} \mathrm{O}$ with two equivalents of $\mathrm{Na}$ (sala-X), made from $\mathrm{H}$ sala-X and $\mathrm{NaOMe}$, as the 
reported procedure did not give the compounds cleanly in our hands. The IR spectroscopic data is consistent with previous reports with an imine stretch observed between $1610-1615 \mathrm{~cm}^{-1}$. UVVis spectroscopic data were recorded in $\mathrm{CH}_{2} \mathrm{Cl}_{2}$ and reveal a band at 629,600 and $615 \mathrm{~nm}$ for 1$\mathrm{Br}$, 1-Me and 1-OMe, respectively (Figure S1). Interestingly, the weaker band at $1000 \mathrm{~nm}$ that is reported in $\mathrm{CHCl}_{3}$, and characteristic of octahedral geometry, is not observed but this may be due to the lower solubility of the compounds in $\mathrm{CH}_{2} \mathrm{Cl}_{2}$.

Recrystallisation of 1- $\mathrm{Br}, 1-\mathrm{Me}$ and 1-OMe from $\mathrm{CH}_{2} \mathrm{Cl}_{2}$ /hexane gives green crystals. Single crystal X-ray crystallography reveals that the compounds belong in the Monoclinic C2/c space group and have a trinuclear structure as shown in Figure 1 (see ESI for full crystallographic details). The asymmetric nature of the sala-X ligand results in one of the $\mathrm{Ni1}$ centres having $\mathrm{N}_{3} \mathrm{O}_{3}$ coordination sphere, while the central $\mathrm{Ni} 2$ has an $\mathrm{O}_{6}$ donor set. The $\mathrm{Ni}(\mathrm{II})$ centres are linked through bridging phenoxides creating face-sharing octahedra. This arrangement is supported by weak $\pi-\pi$ interactions, (3.71 $\AA$ 1-Br; $3.71 \AA$ 1-Me; $3.70 \AA$ 1-OMe, Figure S3 in the ESI) between the central phenyl rings. The $\mathrm{Ni}-\mathrm{N} / \mathrm{O}$ bond lengths are typical of octahedral $\mathrm{Ni}(\mathrm{II})$, and while the bond lengths around $\mathrm{Ni} 2$ are similar to those observed at $\mathrm{Ni1}$, the former is much more distorted as evidenced by the significantly higher $\Sigma$ values. The Ni1-Ni2 distance is virtually identical across the series at $c a$. $2.82 \AA$ suggesting that magnetic communication is likely to be present and efficient across the face-shared bridge.

a)

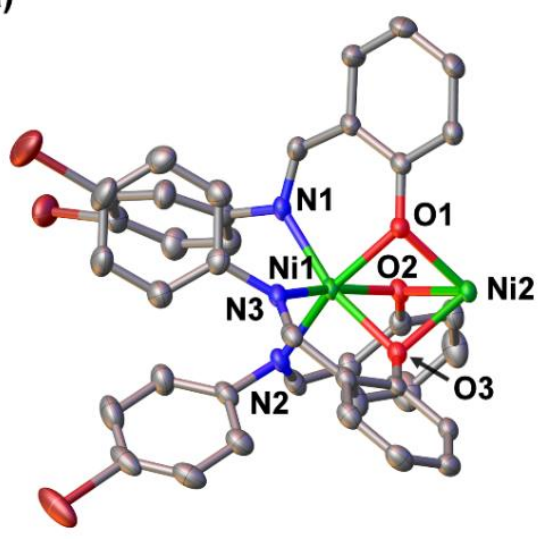

b)

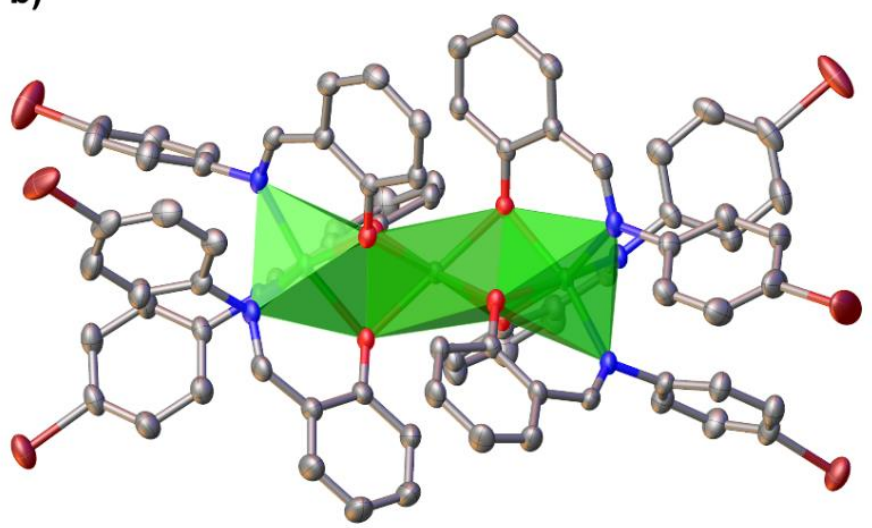

Figure 1 Structure of $\left[\mathrm{Ni}_{3}(\mathrm{sala}-\mathrm{Br})_{6}\right]$ 1-Br showing a) the labelling of the donor atoms in the asymmetric unit of 1-Br and b) face-sharing octahedra with thermal ellipsoids drawn at $50 \%$.

Table 1 Selected bond lengths and angles for 1-Br, 1-Me and 1-OMe $\left(\AA{ }^{\circ},{ }^{\circ}\right.$.

\begin{tabular}{llll}
\hline & $1-\mathrm{Br}$ & $1-\mathrm{Me}$ & $1-\mathrm{OMe}$ \\
\hline $\mathrm{Ni1}-\mathrm{O} 1$ & $2.037(3)$ & $2.0560(10)$ & $2.0475(16)$ \\
$\mathrm{Ni1}-\mathrm{O} 2$ & $2.046(3)$ & $2.0449(10)$ & $2.0454(16)$ \\
$\mathrm{Ni1}-\mathrm{O} 3$ & $2.050(3)$ & $2.0487(10)$ & $2.0546(17)$
\end{tabular}




\begin{tabular}{llll}
$\mathrm{Ni1}-\mathrm{N} 1$ & $2.099(3)$ & $2.0789(12)$ & $2.088(2)$ \\
$\mathrm{Ni1}-\mathrm{N} 2$ & $2.081(3)$ & $2.0790(12)$ & $2.080(2)$ \\
$\mathrm{Ni1}-\mathrm{N} 3$ & $2.070(3)$ & $2.0988(12)$ & $2.082(2)$ \\
$\mathrm{Ni1}-\mathrm{Ni2}$ & $2.8116(6)$ & $2.8248(2)$ & $2.8180(4)$ \\
$\mathrm{Ni2}-\mathrm{O} 1$ & $2.071(3)$ & $2.0632(10)$ & $2.0679(16)$ \\
$\mathrm{Ni2}-\mathrm{O} 2$ & $2.045(3)$ & $2.0554(10)$ & $2.0631(16)$ \\
$\mathrm{Ni2}-\mathrm{O} 3$ & $2.066(3)$ & $2.0680(9)$ & $2.0583(16)$ \\
& & & \\
$\mathrm{Ni1}-\mathrm{O} 1-\mathrm{Ni2}$ & $86.13(10)$ & $86.59(4)$ & $86.43(6)$ \\
$\mathrm{Ni1}-\mathrm{O} 2-\mathrm{Ni2}$ & $87.04(10)$ & $87.09(4)$ & $86.61(6)$ \\
$\mathrm{Ni1}-\mathrm{O} 3-\mathrm{Ni2}$ & $86.16(10)$ & $86.66(4)$ & $86.49(6)$ \\
$\Sigma \mathrm{Ni1}$ & 71.7 & 72.0 & 71.2 \\
$\Sigma \mathrm{Ni2}$ & 147 & 158 & 146 \\
\hline
\end{tabular}

\section{Magnetic studies}

The temperature dependence of the magnetic susceptibility of the three complexes were studied from 1.8-300 K (Figure 2). The compounds exhibit almost identical behaviour and thus we discuss here only 1-Me in detail. At room temperature, the $\chi T$ product is $4.3 \mathrm{~cm}^{3} \mathrm{~K} \mathrm{~mol}^{-1}$, consistent with the expected value for the presence of three isolated $\mathrm{Ni}(I I)$ ions $(S=1)$ with a Landé $g$-factor slight above 2 . When the temperature is lowered, the $\chi T$ product increases continuously to reach a maximum of $6.7 \mathrm{~cm}^{3} \mathrm{~K} \mathrm{~mol}^{-1}$ around $11 \mathrm{~K}$, indicating dominant ferromagnetic interactions within the complex. Below $4 \mathrm{~K}$, the $\chi T$ product drops down to $5.3 \mathrm{~cm}^{3} \mathrm{~K} \mathrm{~mol}^{-1}$ at $1.85 \mathrm{~K}$ suggesting the presence of weak antiferromagnetic interactions, Zeeman depopulation effects and/or a significant magnetic anisotropy. The experimental $(\chi T)_{\max }$ at $11 \mathrm{~K}$ agrees with a $S_{T}=3$ total spin ground state of this trinuclear complex expected for three ferromagnetically arranged $S=1 \mathrm{Ni}$ (II) spins. The $S_{T}=3$ ground state is also supported by the field dependence of the magnetization at low temperatures, which reaches $6.7 \mu_{\mathrm{B}}$ at $7 \mathrm{~T}$ and $1.8 \mathrm{~K}$. The magnetization follows relatively well a simple $S=3$ Brillouin function with $g=2.20(2)$. This indicates that the intrinsic magnetic anisotropy of the $\mathrm{Ni}(I I)$ metal ion is very small as also shown by the almost superposition of the $M$ vs $H / T$ data (see Figures S4-S6 in the ESI). Therefore, antiferromagnetic intermolecular interactions are relevant and dominant at low temperature in this system to explain the decrease of the $\chi T$ product below $11 \mathrm{~K}$. 


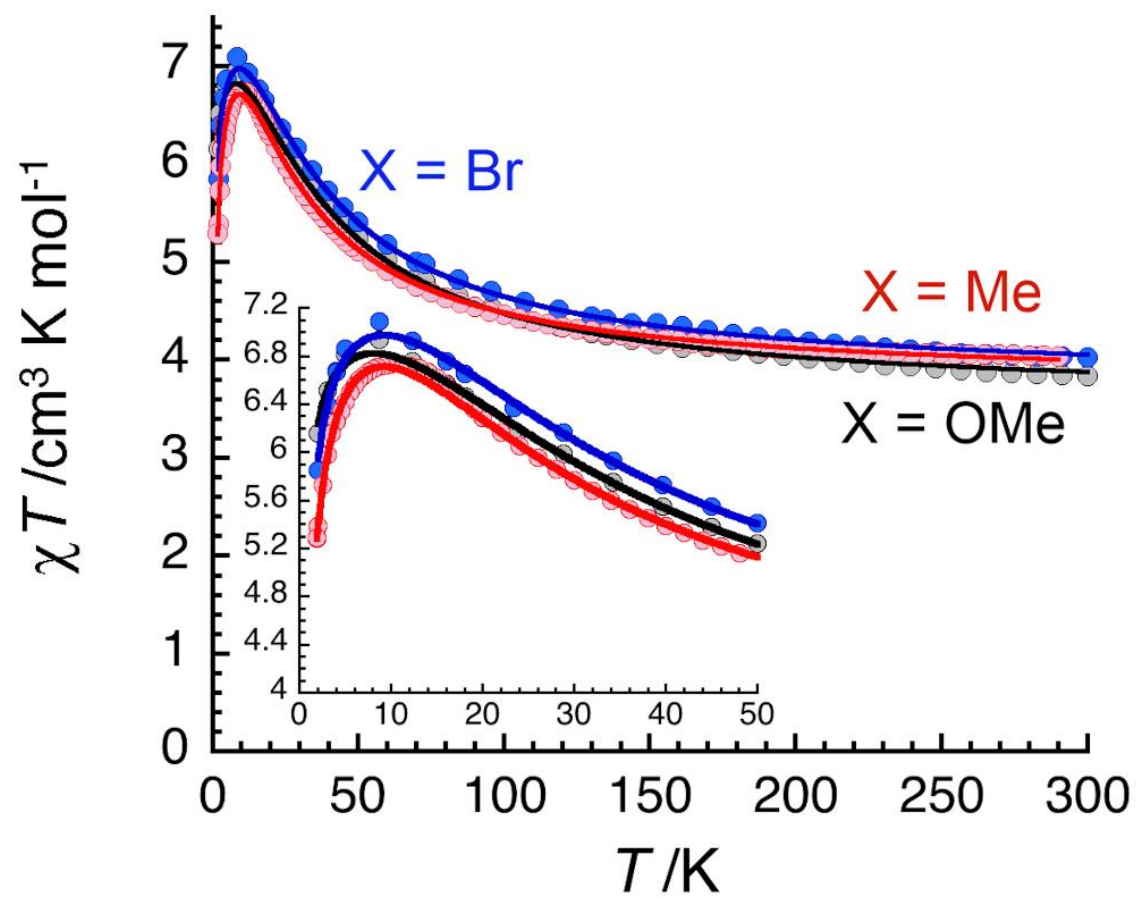

Figure 2 Temperature dependence of the $\chi^{T}$ product (where $\chi$ is the molar magnetic susceptibility that equals $M / H$ per complex) collected in an applied dc magnetic field of $0.1 \mathrm{~T}$ for 1-Br (blue dots), 1-Me (red dots) and 1-OMe (black dots). Inset: View of the low temperature region of the main figure below $50 \mathrm{~K}$. The solid lines are the best fits to the model described in the text.

Considering the structure, the magnetic susceptibility can be modeled using a linear Heisenberg tri-spin $S=1$ model. In keeping with this structural motif, the Heisenberg spin Hamiltonian can be written as follows:

$$
\mathcal{H}=-2 J_{12} \vec{S}_{\mathrm{Ni} 2} \bullet\left(\vec{S}_{\mathrm{Ni} 1 a}+\vec{S}_{\mathrm{Ni} 1 b}\right)
$$

where $J$ is the exchange interactions within the trinuclear complex between adjacent $\mathrm{Ni}(\mathrm{II})$ through tris-phenoxido bridge; $S_{i}$ is the spin operators for each center with $\boldsymbol{S}_{\mathrm{i}}=1$. The application of the van Vleck equation ${ }^{18}$ to the Kambe's vector coupling scheme, ${ }^{19}$ allows us to determine an analytical expression of the magnetic susceptibility in the weak field approximation:

$\chi_{N i_{3}} T=\frac{2 N g^{2} \mu_{B}^{2}}{k_{B} T} \frac{14+5 e^{\frac{-2 J_{12}}{k_{B} T}}+e^{\frac{-4 J_{12}}{k_{B} T}}+6 e^{\frac{-6 J_{12}}{k_{B} T}}+e^{\frac{-10 J_{12}}{k_{B} T}}}{7+5 e^{\frac{-2 J_{12}}{k_{B} T}}+3 e^{\frac{-4 J_{12}}{k_{B} T}}+8 e^{\frac{-6 J_{12}}{k_{B} T}}+e^{\frac{-8 J_{12}}{k_{B} T}}+3 e^{\frac{-10 J_{12}}{k_{B} T}}}$ 
The above model reproduces the experimental data very well down to $20 \mathrm{~K}$ but below $20 \mathrm{~K}$ the introduction of intercomplex magnetic interactions in the frame of the mean-field approximation is required:

$\chi=\frac{\chi_{N i_{3}}}{1-\frac{2 z J^{\prime}}{N g^{2} \mu_{B}^{2}} \chi_{N i_{3}}}$

where $\chi_{\mathrm{Ni3}}$ is the susceptibility of the non-interacting complexes, $z$ the number of nearest neighbors and $J^{\prime}$ is the magnetic interactions between $\mathrm{Ni}_{3}$ complexes. ${ }^{20,21} \mathrm{~A}$ good fit of the data for 1-Me has been achieved using $J_{12} / k_{\mathrm{B}}=+11.4(2) \mathrm{K}, \mathrm{zJ}^{\prime} / \mathrm{k}_{\mathrm{B}}=-0.097(2) \mathrm{K}$ and $g_{\mathrm{Ni}}=2.23(2)$, while the parameters obtained in the cases of 1- $\mathbf{B r}$ and 1-OMe are given in Table 2. These values are broadly comparable with a range of $\mathrm{Ni}(\mathrm{II})$ trinuclear complexes. ${ }^{22-24}$ As mentioned before, the sign of the magnetic interactions implies that this complex possesses an $S_{T}=3$ spin ground state. This result is consistent with the field dependence shown in supporting information, that is very close to a $S=3$ Brillouin function. Caution is needed regarding $z J^{\prime}$ as it could phenomenologically contain the contribution from the $\mathrm{Ni}$ (II) magnetic anisotropy. While a numerical model considering only the local magnetic anisotropy is unable to reproduce the experimental data (using PHI), ${ }^{25}$ a model considering the local magnetic anisotropy in addition to intermolecular interactions does not significantly improve the theory/experiment agreement.

Table 2 Magnetic fitting parameters for 1-Br, 1-Me and 1-OMe.

\begin{tabular}{lllll}
\hline & $\boldsymbol{J}_{12} / \mathbf{k}_{\mathrm{B}}(\mathrm{K})$ & $\mathbf{z} \mathbf{J}^{\prime} / \mathbf{k}_{\mathrm{B}}(\mathrm{K})$ & $\boldsymbol{g}_{\mathrm{Ni}}$ & $\mathbf{N i - O}-\mathbf{N i}_{\text {ave }}\left({ }^{\circ}\right)$ \\
\hline 1-Br & $+13.9(5)$ & $-0.065(4)$ & $2.24(5)$ & 86.44 \\
1-Me & $+11.4(2)$ & $-0.097(2)$ & $2.23(2)$ & 86.77 \\
1-OMe & $+14.7(5)$ & $-0.036(4)$ & $2.18(5)$ & 86.51 \\
\hline
\end{tabular}

\section{Theoretical Studies}

Calculations based on Density Functional Theory provide an excellent estimation of the exchange coupling constants in polynuclear transition metal complexes despite the very small energy differences involved. ${ }^{26,27}$ Detailed description of the computational procedure employed to calculate the exchange coupling constants in polynuclear complexes has been previously provided by some of us. ${ }^{28,29}$ The calculations were performed using Gaussian16 B.01 version ${ }^{30}$ with the B3LYP functional ${ }^{31}$ and an all electron basis set (Def2TZV). ${ }^{32}$ For the studied symmetric trinuclear $\mathrm{Ni}$ (II) complexes, there are two intramolecular exchange interactions (see Table 3), and we employed three spin configurations to estimate these two $J$ values: the high spin solution ( $S$ $=3$ ) and two $S=1$ wavefunctions obtained with the spin inversion of the terminal or central nickel 
center. The magnetostructural correlation with the calculated $J_{12}$ values shows the expected trend being more ferromagnetic coupling when the Ni-O-Ni angle becomes smaller. ${ }^{33}$ This fact is due to the well-known dependence, complexes with smaller bridging $\mathrm{Ni}-\mathrm{O}-\mathrm{Ni}$ angles have a weaker antiferromagnetic term. At the error bar of the experimental and theoretical data, the calculated values are in good agreement with the experimentally fitted parameters (Tables 2 and 3). The next-nearest neighbour interactions are weak and antiferromagnetic, as usual, but they do not follow a clear tendency with the Ni-O-Ni angle.

Table 3 DFT calculated intramolecular exchange coupling constants $\left(J_{12}\right.$ next- and $J_{13}$ next-nearest neighbour interaction) for three trinuclear $\mathrm{Ni}(\mathrm{II})$ complexes using the experimental X-ray structures.

\begin{tabular}{llll}
\hline & $\boldsymbol{J}_{12} / \mathbf{k}_{\mathrm{B}}(\mathrm{K})$ & $\boldsymbol{J}_{13} / \mathbf{k}_{\mathrm{B}}(\mathrm{K})$ & $\mathbf{N i - O}-\mathrm{Ni}_{\text {ave }}\left(^{\circ}\right)$ \\
\hline 1-Br & +12.1 & -0.042 & 86.44 \\
1-Me & +11.6 & -0.034 & 86.77 \\
1-OMe & +11.8 & -0.030 & 86.51 \\
\hline
\end{tabular}

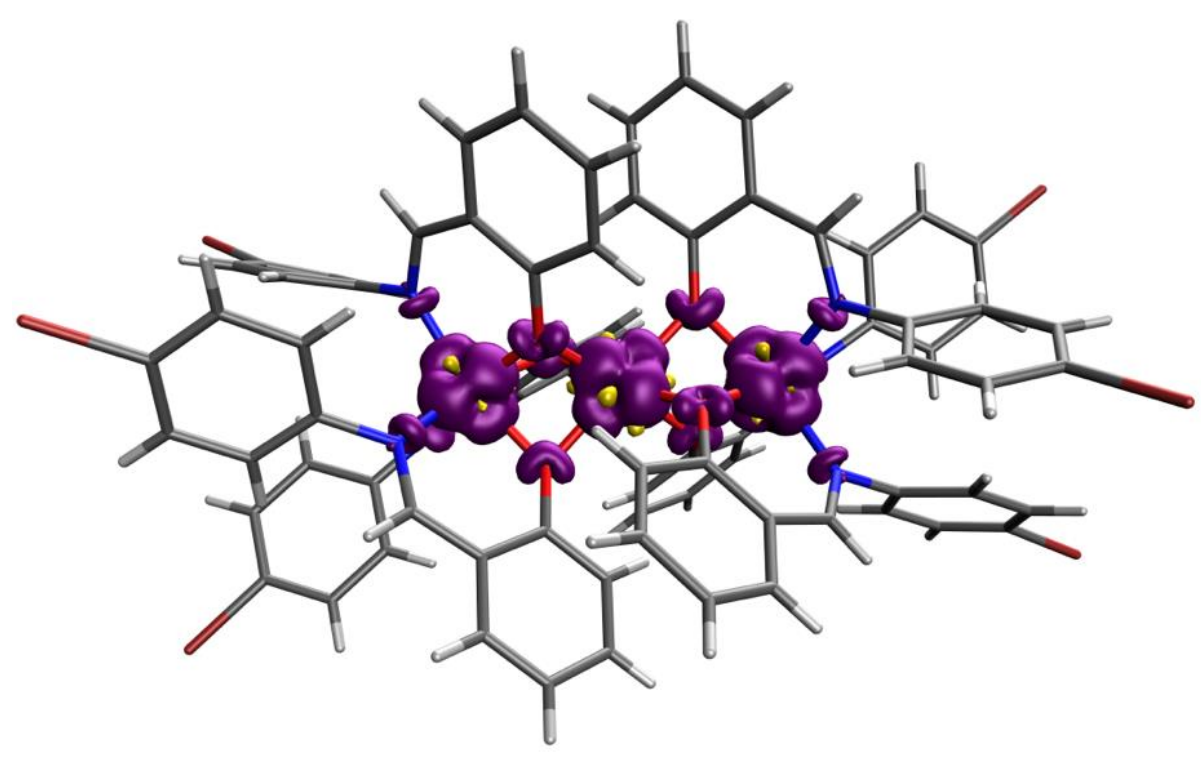

Figure 3 Spin population map corresponding the $S=3$ ground state of 1- $\mathrm{Br}$ calculated with the B3LYP method. The purple and yellow colors indicate positive and negative values, respectively. The isosurface corresponds to $0.14 \mathrm{e}^{-3}$.

As an example, the spin density map of the $S=3$ ground state of 1-Br system is represented in Figure 3. As the coupling is ferromagnetic the main contributions in the three nickel atoms are positive due to the unpaired electrons in the $e_{g}$ orbitals. Consequently, the positive lobes are 
oriented towards the coordinated ligand atom. A small negative population in the metal atoms (see yellow lobes in Figure 3 ) are due to the spin polarization mechanism ${ }^{34}$ of the non-bonding $t_{2 g}$ orbitals. In the atoms of the ligands corresponding the first coordination sphere, the spin delocalization mechanism is predominant. ${ }^{35}$ This is due to the antibonding nature of the $e_{g}$ orbitals bearing the unpaired electrons in the metal ion with a large mixing with the ligand orbitals. Thus, positive densities are also found on the atoms of the first coordination sphere.

\section{Conclusions}

To conclude, we have definitively confirmed that the $\mathrm{Ni}(\mathrm{II})$ salicylaldimine complexes with parasubstituents exist as face-sharing trinuclear $\mathrm{Ni}(\mathrm{II})$ complexes in the solid state. In these complexes, the magnetic susceptibility measurements demonstrate that the $\mathrm{Ni}(\mathrm{II})$ spins are ferromagnetically coupled, consistent with the $\mathrm{Ni}-\mathrm{O}-\mathrm{Ni}$ angle of $86-87^{\circ}$. DFT results confirm the ferromagnetic nature of the exchange interactions. Moreover, the calculated $J_{12}$ values indicate the expected magnetostructural trend with the smaller bridging $\mathrm{Ni}-\mathrm{O}-\mathrm{Ni}$ angle showing stronger ferromagnetic coupling. Preliminary studies suggest that these trinuclear $\mathrm{Ni}(\mathrm{II})$ complexes may be used in the construction of more complex systems and these will be reported in due course.

\section{Acknowledgements}

We thank the Thailand Science Research and Innovation Fund (Contract No. WU-FF 64101) for funding this research. The National Science Technology and Innovation Policy Office for Integrated Research and Innovation Plan (Grant No. 256113A3050001) is thanked for funds to purchase an X-ray diffractometer. We also thank the CNRS, the University of Bordeaux, the Région Nouvelle Aquitaine, the GdR MCM-2: Magnétisme et Commutation Moléculaires, and Quantum Matter Bordeaux for financial support. We also thank the Spanish Ministerio de Ciencia e Innovación, (PGC2018-093863-B-C21 and MDM-2017-0767) for financial support and CSUC for computer facilities. We also thank the Australian Research Council for a Discovery grant. (Grant No. DP170100034).

\section{References}

1 R. Hernández-Molina and A. Mederos, in Comprehensive Coordination Chemistry II, eds. J. A. McCleverty and T. J. Meyer, 2003, pp. 411-446.

2 C. Wang, S. Friedrich, T. R. Younkin, R. T. Li, R. H. Grubbs, D. A. Bansleben and M. W. Day, Organometallics, 1998, 7333, 3149-3151.

3 T. R. Younkin, E. F. Connor, J. I. Henderson, S. K. Friedrich, R. H. Grubbs and D. A. Bansleben, Science, 2000, 287, 460-463.

4 E. F. Connor, T. R. Younkin, J. I. Henderson, A. W. Waltman and R. H. Grubbs, Chem. Commun., 2003, 2272-2273.

5 I. Göttker-Schnetmann, P. Wehrmann, C. Röhr and S. Mecking, Organometallics, 2007, 


\section{6, 2348-2362.}

6 P. Kenyon, M. Wo and S. Mecking, J. Am. Chem. Soc., 2018, 140, 6685-6689.

7 M. Schnitte, J. S. Scholliers, K. Riedmiller and S. Mecking, Angew. Chem. Int. Ed., 2020, 59, 3258-3263.

8 H.-L. Mu, W.-P. Ye, D.-P. Song and Y.-S. Li, Organometallics, 2010, 29, 6282-6290.

9 M. Janeta, J. X. Heidlas, O. Daugulis and M. Brookhart, Angew. Chem. Int. Ed., 2021, 60, 4566-4569.

10 R. H. Holm and K. Swaminathan, Inorg. Chem., 1962, 1, 599-607.

11 R. H. Holm and K. Swaminathan, Inorg. Chem., 1963, 2, 181-186.

12 P. C. Percy and D. A. Thorton, Inorg. Nucl. Chem. Lettters, 1971, 7, 599-604.

13 G. C. Percy and D. A. Thorton, J. Inorg. Nucl. Chem., 1972, 34, 3357-3367.

14 R. H. Holm, G. W. Everett and A. Chakravorty, in Progress in Inorganic Chemistry, John Wiley \& Sons, Ltd, 1966, pp. 83-214.

15 G. M. Sheldrick, Acta Crystallogr. Sect. A Found. Crystallogr., 2015, 71, 3-8.

16 G. M. Sheldrick, Acta Crystallogr. Sect. C Struct. Chem., 2015, 71, 3-8.

17 O. V. Dolomanov, L. J. Bourhis, R. J. Gildea, J. A. K. Howard and H. Puschmann, J. Appl. Cryst., 2009, 42, 339-42.

18 J. H. van Vleck, The Theory of Electric and Magnetic Susceptibility, Oxford University Press, London, 1932.

19 K. Kambe, J. Phys. Soc. Jpn., 1950, 5, 48-51.

20 B. E. Myers, L. Berger and S. Friedberg, J. Appl. Phys., 1969, 40, 1149.

21 C. J. O'Connor, in Prog. Inorg. Chem., John Wiley \& Sons, Ltd, 1982, vol. 29, pp. 203-283.

22 H. Ohta, K. Harada, K. Irie, S. Kashino, T. Kambe, G. Sakane, T. Shibahara, S. Takamizawa, W. Mori, M. Nonoyama, M. Hirotsu and M. Kojima, Chem. Lett., 2001, 2, 842-843.

23 T. Kobayashi, T. Yamaguchi, H. Ohta, Y. Sunatsuki, M. Kojima, N. Re, M. Nonoyama and N. Matsumoto, Chem. Commun., 2006, 1950-1952.

24 P. Seth, A. Figuerola, J. Jover, E. Ruiz and A. Ghosh, Inorg. Chem., 2014, 53, 9296-9305.

25 N. F. Chilton, R. P. Anderson, L. D. Turner, A. Soncini, K. S. Murray, S. J. Comput. Chem. 34 (2013) 1164-1175; N.F. Chilton, PHI User Manual v2.1 (2015).

26 E. Ruiz, in Comprehensive Inorganic Chemistry II (Second Edition), eds. J. Reedijk and K. Poeppelmeier, Elsevier, Amsterdam, 2013, pp. 501-549.

27 E. Ruiz, S. Alvarez, A. Rodríguez-Fortea, P. Alemany, Y. Pouillon and C. Massobrio in Electronic Structure and Magnetic Behavior in Polynuclear Transition-metal Compounds, Vol. 2 Eds.: J. S. Miller and M. Drillon), Wiley-VCH, Weinheim, 2001, pp. 227-279

28 E. Ruiz, S. Alvarez, J. Cano and V. Polo, J. Chem. Phys., 2005, 123, 164110

29 E. Ruiz, A. Rodríguez-Fortea, J. Cano, S. Alvarez, P. Alemany, J. Comp. Chem., 2003, 24, 982-989.

30 Gaussian 16, Revision B.01, M. J. Frisch, G. W. Trucks, H. B. Schlegel, G. E. Scuseria, M. A. Robb, J. R. Cheeseman, G. Scalmani, V. Barone, G. A. Petersson, H. Nakatsuji, X. Li, M. Caricato, A. V. Marenich, J. Bloino, B. G. Janesko, R. Gomperts, B. Mennucci, H. P. Hratchian, J. V. Ortiz, A. F. Izmaylov, J. L. Sonnenberg, D. Williams-Young, F. Ding, F. Lipparini, F. Egidi, J. Goings, B. Peng, A. Petrone, T. Henderson, D. Ranasinghe, V. G. Zakrzewski, J. Gao, N. Rega, G. Zheng, W. Liang, M. Hada, M. Ehara, K. Toyota, R. Fukuda, J. Hasegawa, M. Ishida, T. Nakajima, Y. Honda, O. Kitao, H. Nakai, T. Vreven, K. Throssell, 
J. A. Montgomery, Jr., J. E. Peralta, F. Ogliaro, M. J. Bearpark, J. J. Heyd, E. N. Brothers, K. N. Kudin, V. N. Staroverov, T. A. Keith, R. Kobayashi, J. Normand, K. Raghavachari, A. P. Rendell, J. C. Burant, S. S. Iyengar, J. Tomasi, M. Cossi, J. M. Millam, M. Klene, C. Adamo, R. Cammi, J. W. Ochterski, R. L. Martin, K. Morokuma, O. Farkas, J. B. Foresman, and D. J. Fox, Gaussian, Inc., Wallingford CT, 2016.

31 A. D. Becke, J. Chem. Phys., 1993, 98, 5648-5652.

32 F. Weigend, R. Ahlrichs Phys. Chem. Chem. Phys., 2005, 7, 3297-305.

33 C. Loose, E. Ruiz, B. Kersting and J. Kortus, Chem. Phys. Lett., 2008, 452, 38-43.

34 J. Cano, E. Ruiz, S. Alvarez and M. Verdaguer, Comments on Inorg. Chem., 1998, 20, 2756.

35 E. Ruiz, J. Cirera and S. Alvarez, Coord. Chem. Rev., 2005, 249, 2649-2660. 


\section{TOC Entry}

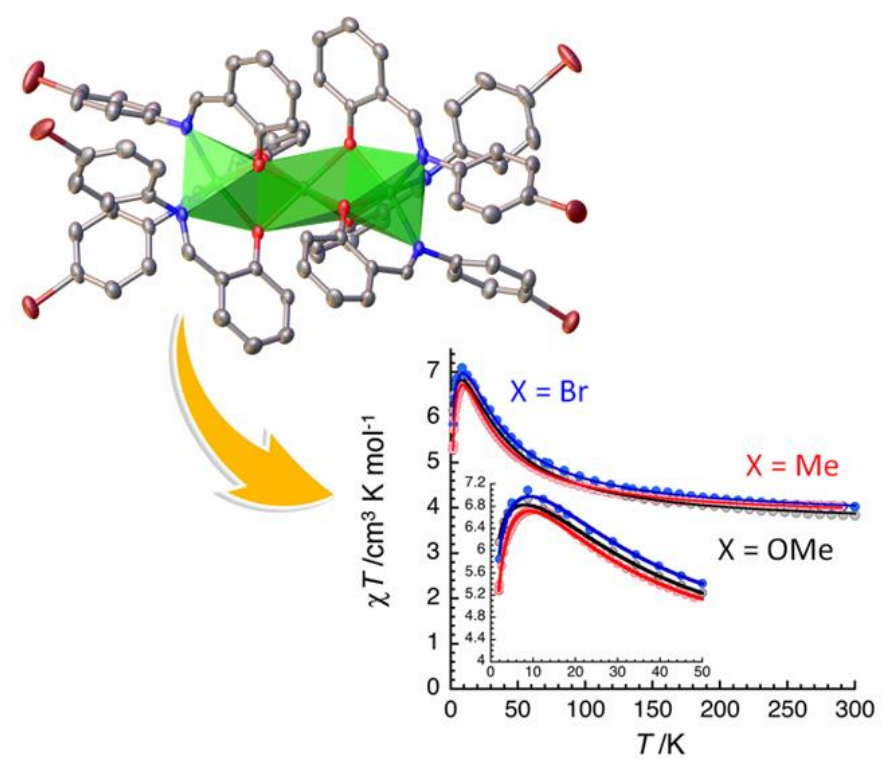

The trinuclear structure of three $\mathrm{Ni}(\mathrm{II})$ salicylaldiminates is convincing demonstrated for the first time with ferromagnetic communication aided by the phenoxide bridges. 